\title{
On Riesz Mean Inequalities for Subelliptic Laplacian
}

\author{
Gao Jia, Jianming Wang, Ya Xiong \\ College of Science, University of Shanghai for Science and Technology, Shanghai, China \\ E-mail: gaojia79@yahoo.com.cn
}

Received March 23, 2011; revised April 6, 2011; accepted April 13, 2011

\begin{abstract}
In this paper, we mainly focus on the Riesz means of eigenvalues of the subelliptic Laplacian on the Heisenberg group $H^{n}$. We establish a trace formula of associated eigenvalues, then we prove differential inequalities, difference inequalities and monotonicity formulas for the Riesz means of eigenvalues of the subelliptic Laplacian.
\end{abstract}

Keywords: Heisenberg Group, Riesz Mean, Subelliptic Laplacian

\section{Introduction}

Until now, the eigenvalue estimations of Laplacian on the bounded Euclidean domain have been extensively studied (see [1-5]). In recent years, some academics have already started to pay attention to the Heisenberg group $H^{n}$, such as P. Levy-Bmhl [6], D. Müller [7], P. C. Niu [8], G. Jia [9] and so on.

The Heisenberg group plays an important role in several branches of mathematics such as representation theory, harmonic analysis, several complex variables, partial differential equations and quantum mechanics. In the past decades research on Heisenberg sub-Laplacian has achieved considerable progress. But the problem of the invariant differential operator eigenvalue for the Heisenberg group, did not be studied deeply.

In this paper, the Riesz mean inequalities of eigenvalues for the subelliptic Laplacian is treated. And some differential inequalities and difference inequalities are established.

The outline of the paper is as follows. In Section 2, we first recall some definitions and the lemmas that will be used in the following, and then establish the trace formula of eigenvalues. Main results and their proofs will be given in Section 3.

\section{Preliminaries and Trace Formula}

Let $H^{n}$ denote Heisenberg group which is a Lie group that has algebra $g=R^{2 n+1}$, with a nonabelian group law

$$
\begin{aligned}
& \left(x_{1}, y_{1}, t_{1}\right) \cdot\left(x_{2}, y_{2}, t_{2}\right) \\
& =\left(x_{1}+x_{2}, y_{1}+y_{2}, t_{1}+t_{2}+2\left(y_{2} x_{1}-x_{2} y_{1}\right)\right)
\end{aligned}
$$

For every $u_{1}=\left(x_{1}, y_{1}, t_{1}\right), u_{2}=\left(x_{2}, y_{2}, t_{2}\right) \in H^{n}$
The Lie algebra is generated by the left invariant vector fields

$$
\begin{aligned}
& X_{i}=\frac{\partial}{\partial x_{i}}+2 y_{i} \frac{\partial}{\partial t} \quad Y_{i}=\frac{\partial}{\partial y_{i}}-2 x_{i} \frac{\partial}{\partial t}, \\
& i=1,2, \cdots, n,
\end{aligned}
$$

And $T=\frac{\partial}{\partial t}$. We set

$$
\nabla_{H^{n}} u=\left(X_{1}(u), \cdots, X_{n}(u), Y_{1}(u), \cdots, Y_{n}(u)\right) .
$$

Remark 2.1

It is easy to see that $X_{i}, Y_{i}, T$ are skew symmetric operators, and

$$
\left[X_{i}, Y_{j}\right]=-4 T \delta_{i j},\left[X_{i}, T\right]=\left[Y_{i}, T\right]=0,
$$

where $[X, Y]$ denotes the standard commutator $X Y-Y X$.

\section{Definition 2.1 [10].}

The subelliptic Laplacian is defined as

$$
\begin{aligned}
\Delta_{H^{n}}= & \sum_{i=1}^{n}\left(X_{i}^{2}+Y_{i}^{2}\right)=\sum_{i=1}^{n}\left[\frac{\partial^{2}}{\partial x_{i}^{2}}+\frac{\partial^{2}}{\partial y_{i}^{2}}-4 x_{i} \frac{\partial^{2}}{\partial y_{i} \partial t}\right. \\
& \left.+4 y_{i} \frac{\partial^{2}}{\partial x_{i} \partial t}+4\left(x_{i}^{2}+y_{i}^{2}\right) \frac{\partial^{2}}{\partial t^{2}}\right]
\end{aligned}
$$

By the definitions and properties of $X_{i}$ and $Y_{i}$, it is easy to see that $\Delta_{H^{n}}$ is invariant with respect to lefttranslations.

Let us concern with the eigenvalue problem

$$
\begin{cases}\Delta_{H^{n}} u=-\lambda u, & \text { in } \Omega, \\ u=0, & \text { on } \partial \Omega .\end{cases}
$$

where $\Omega$ is a bounded domain of the Heisenberg group 
$H^{n}$ with smooth boundary. By [8], we see that the Dirichlet problem (2.4) has a discrete spectrum on a Hilbert space with Inner product denoted $\langle\cdot, \cdot\rangle$, and its eigenvalues by $0<\lambda_{1} \leq \lambda_{2} \leq \cdots \leq \lambda_{k} \cdots$ with

$\lim _{k \rightarrow \infty} \lambda_{k}=+\infty$ and orthonormalize its eigenfunctions

$u_{1}, u_{2}, \cdots \in S_{0}^{1,2}(\Omega)$ so that

$$
\left\langle u_{i}, u_{j}\right\rangle=\int_{\Omega} u_{i} u_{j} \mathrm{~d} x \mathrm{~d} y \mathrm{~d} t=\delta_{i j}, \forall i, j \geq 1 .
$$

Here, $S^{1,2}(\Omega)$ denotes the Hilbert space of the functions $u \in L^{2}(\Omega)$ such that $X_{i}(u), Y_{i}(u) \in L^{2}(\Omega)$, and $S_{0}^{1,2}$ denotes the closure of $C_{0}^{\infty}(\Omega)$.

For the sake of simplicity, let $L$ be a form

$$
L=-\Delta_{H^{n}}=-\sum_{\alpha=1}^{n}\left(X_{\alpha}^{2}+Y_{\alpha}^{2}\right) .
$$

There will be a distinguished subset $J_{j}=\left\{\lambda_{1}, \lambda_{2}, \cdots, \lambda_{j}\right\}$ of the spectrum of $L$, $J_{j}^{c}=\left\{\lambda_{1}, \cdots, \lambda_{j}, \cdots\right\} \backslash\left\{\lambda_{1}, \cdots, \lambda_{j}\right\}$ is the complement of $J_{j}$, and $P_{J_{j}}, P_{J_{j}^{c}}$ will be the corresponding spectral projections. We shall be interested in traces of $P_{J_{j}} f(L)$, where $f\left(\lambda_{j}\right)$ is any function defined on the spectrum of $L$.

\section{Definition 2.2.}

If $\left\{\lambda_{k}\right\}_{k=1}^{\infty}$ is an increasing sequence of real numbers, for $z \geq 0$, the Riesz mean of order $\sigma>0$ of $\left\{\lambda_{k}\right\}$ can be defined as $[11,12]$

$$
R_{\sigma}(z)=\sum_{k=1}^{\infty}\left(z-\lambda_{k}\right)_{+}^{\sigma}
$$

where $\left(z-\lambda_{k}\right)_{+}:=\max \left(0, z-\lambda_{k}\right)$ is the ramp function.

\section{Definition 2.3.}

Two symmetric operators $V_{\alpha}, W_{\alpha}$ are defined as $V_{\alpha} u=x_{\alpha} u, W_{\alpha} u=y_{\alpha} u,(\alpha=1, \cdots, n)$.

\section{Remark 2.2.}

$$
\begin{gathered}
{\left[X_{\alpha}, V_{\beta}\right]= \begin{cases}I, & \alpha=\beta \\
0, & \alpha \neq \beta\end{cases} } \\
{\left[Y_{\alpha}, W_{\beta}\right]= \begin{cases}I, & \alpha=\beta \\
0, & \alpha \neq \beta\end{cases} } \\
{\left[X_{\alpha}, W_{\beta}\right]=0\left[Y_{\alpha}, V_{\beta}\right]=0,}
\end{gathered}
$$

here $I$ is an identity operator. In fact, we have

$$
\begin{aligned}
{\left[X_{\alpha}, V_{\alpha}\right] u=} & \frac{\partial}{\partial x_{\alpha}}\left(x_{\alpha} u\right)+2 y_{\alpha} \frac{\partial}{\partial t}\left(x_{\alpha} u\right) \\
& -x_{\alpha}\left(\frac{\partial u}{\partial x_{\alpha}}+2 y_{\alpha} \frac{\partial u}{\partial t}\right)=u
\end{aligned}
$$

i.e. $\left[X_{\alpha}, V_{\alpha}\right]=I$, similarly $\left[Y_{\alpha}, W_{\alpha}\right]=I$, and so on.

\section{Theorem 2.1.}

Let $\lambda_{j}$ and $u_{j}$ be eigenvalues and $L^{2}$-normalized eigenfunctions of the subelliptic Laplacian. Let

$$
T_{\alpha j m}:=\left|\left\langle X_{\alpha} u_{j}, u_{m}\right\rangle\right|^{2}+\left|\left\langle Y_{\alpha} u_{j}, u_{m}\right\rangle\right|^{2}
$$

for $j, m=1,2, \cdots$ and $\alpha=1, \cdots, n$. Then for each fixed $\alpha$,

$$
\begin{aligned}
\sum_{\lambda_{j} \in J_{j}} f\left(\lambda_{j}\right)= & -\sum_{\substack{\lambda_{j}, \lambda_{m} \in J_{j} \\
\lambda_{j} \neq \lambda_{m}}} \frac{f\left(\lambda_{j}\right)-f\left(\lambda_{m}\right)}{\lambda_{j}-\lambda_{m}} T_{\alpha j m} \\
& +2 \sum_{\substack{\lambda_{j} \in J_{j} \\
\lambda_{q} \in J_{j}^{c}}} \frac{f\left(\lambda_{j}\right)}{\lambda_{q}-\lambda_{j}} T_{\alpha j q}
\end{aligned}
$$

Specialized to $f\left(\lambda_{j}\right)=\left(z-\lambda_{j}\right)_{+}^{\sigma}$, we can obtain

$$
\begin{aligned}
R_{\sigma}(z)= & \sum_{j, m: \lambda_{j} \neq \lambda_{m}} \frac{\left(z-\lambda_{j}\right)_{+}^{\sigma}-\left(z-\lambda_{m}\right)_{+}^{\sigma}}{\lambda_{m}-\lambda_{j}} T_{\alpha j m} \\
& +2 \sum_{j, q: \lambda_{j} \leq z<\lambda_{q}} \frac{\left(z-\lambda_{j}\right)_{+}^{\sigma}}{\lambda_{q}-\lambda_{j}} T_{\alpha j q}
\end{aligned}
$$

To derive out Theorem 2.1, we need the following lemma.

Lemma 2.1 [6].

Let $0<x<y$ and $\sigma \geq 0$. Then

$$
\frac{y^{\sigma}-x^{\sigma}}{y-x} \leq C_{\sigma}\left(y^{\sigma-1}+x^{\sigma-1}\right)
$$

where

$$
C_{\sigma}:=\left\{\begin{array}{l}
\frac{\sigma}{2}, \text { if } 0 \leq \sigma<1 \text { or } 2 \leq \sigma<\infty \\
1, \text { if } 1 \leq \sigma \leq 2
\end{array} .\right.
$$

Proof of Theorem 2.1.

Observe that because $\left\{u_{j}\right\}_{j=1}^{\infty}$ is a complete orthonormal system,

$$
T_{j}=\sum_{\alpha=1}^{n} \sum_{m=1}^{\infty} T_{\alpha j m}=\sum_{\alpha=1}^{n} \sum_{m=1}^{\infty}\left|\left\langle X_{\alpha} u_{j}, u_{m}\right\rangle\right|^{2}+\left|\left\langle Y_{\alpha} u_{j}, u_{m}\right\rangle\right|^{2}
$$

According to [10] the formal commutator identity $[A, B C]=B[A, C]+[A, B] C$, we have $\left[L, V_{\alpha}\right]=\left[-X_{\alpha} \cdot X_{\alpha}-Y_{\alpha} \cdot Y_{\alpha}, V_{\alpha}\right]=-2 X_{\alpha}$. Similarly, we get $\left[L, W_{\alpha}\right]=-2 Y_{\alpha}$.

Thus

$$
\begin{aligned}
T_{j} & =\frac{1}{4} \sum_{\alpha=1}^{n} \sum_{m=1}^{\infty}\left|\left\langle\left[L, V_{\alpha}\right] u_{j}, u_{m}\right\rangle\right|^{2}+\left|\left\langle\left[L, W_{\alpha}\right] u_{j}, u_{m}\right\rangle\right|^{2} \\
& =\int_{\Omega}\left|\nabla_{H^{n}} u_{j}\right|^{2}=\lambda_{j}
\end{aligned}
$$

By [2], we obtain

$\operatorname{tr}\left(P_{J_{j}} f(L)\right)=\operatorname{tr}\left(P_{J_{j}} f(L)\left[X_{\alpha}, V_{\alpha}\right]\right)$, and 


$$
\begin{aligned}
\operatorname{tr}\left(P_{J_{j}} f(L)\right)= & -2 \sum_{\substack{\lambda_{j}, \lambda_{m} \in J_{j} \\
\lambda_{j} \neq \lambda_{m}}} \frac{f\left(\lambda_{j}\right)-f\left(\lambda_{m}\right)}{\lambda_{j}-\lambda_{m}}\left|\left\langle X_{\alpha} u_{j}, u_{m}\right\rangle\right|^{2} \\
& +4 \sum_{\substack{\lambda_{j} \in J_{j} \\
\lambda_{q} \in J_{j}^{c}}} \frac{f\left(\lambda_{j}\right)}{\lambda_{q}-\lambda_{j}}\left|\left\langle X_{\alpha} u_{j}, u_{q}\right\rangle\right|^{2}
\end{aligned}
$$
then

And similarly $\operatorname{tr}\left(P_{J_{j}} f(L)\right)=\operatorname{tr}\left(P_{J_{j}} f(L)\left[Y_{\alpha}, W_{\alpha}\right]\right)$,

$$
\begin{aligned}
\operatorname{tr}\left(P_{J_{j}} f(L)\right)= & -2 \sum_{\substack{\lambda_{j}, \lambda_{m} \in J_{j} \\
\lambda_{j} \neq \lambda_{m}}} \frac{f\left(\lambda_{j}\right)-f\left(\lambda_{m}\right)}{\lambda_{j}-\lambda_{m}}\left|\left\langle Y_{\alpha} u_{j}, u_{m}\right\rangle\right|^{2} \\
& +4 \sum_{\substack{\lambda_{j} \in J_{j} \\
\lambda_{q} \in J_{j}^{C}}} \frac{f\left(\lambda_{j}\right)}{\lambda_{q}-\lambda_{j}}\left|\left\langle Y_{\alpha} u_{j}, u_{q}\right\rangle\right|^{2}
\end{aligned}
$$

Summing of the (2.10) and (2.11), we obtain

$$
\begin{aligned}
2 \operatorname{tr}\left(P_{J_{j}} f(L)\right)= & -2 \sum_{\substack{\lambda_{j}, \lambda_{m} \in J_{j} \\
\lambda_{j} \neq \lambda_{m}}} \frac{f\left(\lambda_{j}\right)-f\left(\lambda_{m}\right)}{\lambda_{j}-\lambda_{m}} T_{\alpha j m} \\
& +4 \sum_{\substack{\lambda_{j} \in J_{j} \\
\lambda_{q} \in J_{j}^{c}}} \frac{f\left(\lambda_{j}\right)}{\lambda_{q}-\lambda_{j}} T_{\alpha j q}
\end{aligned}
$$

Since $\sum_{\lambda_{j} \in J_{j}} f\left(\lambda_{j}\right)=\operatorname{tr}\left(P_{J_{j}} f(L)\right)$, we get (2.6), and the proof of the Theorem is completed.

\section{Riesz Means Inequalities}

In this section, we derive differential inequalities and difference inequalities for the Riesz means $R_{\sigma}(z)=\sum_{k=1}^{\infty}\left(z-\lambda_{k}\right)_{+}^{\sigma}$. Here $\left\{\lambda_{k}\right\}_{k=1}^{\infty}$ are ordered eigenvalues of the subelliptic Laplacian on a bounded domain.

Theorem 3.1.

For $0<\sigma \leq 2$ and $z \geq \lambda_{1}$, then we have

$$
\begin{gathered}
R_{\sigma-1}(\mathrm{z}) \geq\left(1+\frac{n}{2}\right) \frac{1}{\mathrm{z}} R_{\sigma}(\mathrm{z}), \\
R_{\sigma}^{\prime}(\mathrm{z}) \geq\left(1+\frac{n}{2}\right) \frac{\sigma}{\mathrm{z}} R_{\sigma}(\mathrm{z}),
\end{gathered}
$$

and $\frac{R_{\sigma}(z)}{z^{\sigma\left(1+\frac{n}{2}\right)}}$ is a nondecreasing function with respect to $Z$.

For $\sigma \geq 2$ and $z \geq \lambda_{1}$, then we have

$$
\begin{gathered}
R_{\sigma-1}(z) \geq\left(1+\frac{n}{\sigma}\right) \frac{1}{z} R_{\sigma}(z) \\
R_{\sigma}^{\prime}(z) \geq(\sigma+n) \frac{1}{z} R_{\sigma}(z)
\end{gathered}
$$

and $\frac{R_{\sigma}(z)}{z^{\sigma+n}}$ is a nondecreasing function with respect to $z$.

Proof. Let the first term on the right of (2.7) be

$$
G(\sigma, \alpha, z):=\sum_{\substack{j, m: \lambda_{j}, \lambda_{m} \leq z \\ \lambda_{j} \neq \lambda_{m}}} \frac{\left(z-\lambda_{j}\right)_{+}^{\sigma}-\left(z-\lambda_{m}\right)_{+}^{\sigma}}{\lambda_{m}-\lambda_{j}} T_{\alpha j m}
$$

By Lemma 2.1, the expression can be simplified to

$$
\begin{aligned}
G(\sigma, \alpha, z):= & \sum_{\substack{j, m: \lambda_{j}, \lambda_{m} \leq z \\
\lambda_{j} \neq \lambda_{m}}} \frac{\left(z-\lambda_{j}\right)_{+}^{\sigma}-\left(z-\lambda_{m}\right)_{+}^{\sigma}}{\lambda_{m}-\lambda_{j}} T_{\alpha j m} \\
\leq & 2 C_{\sigma} \sum_{j, m: \lambda_{j} \leq z} \sum_{m=1}^{\infty}\left(z-\lambda_{j}\right)_{+}^{\sigma-1} T_{\alpha j m} \\
& -2 C_{\sigma} \sum_{j, m: \lambda_{j} \leq z<\lambda_{m}}\left(z-\lambda_{j}\right)_{+}^{\sigma-1} T_{\alpha j m}
\end{aligned}
$$

By symmetry in $j \leftrightarrow m$, extending the sum to all $m$ subtracting the same quantity from the final term in (2.7), we find

$$
\begin{aligned}
R_{\sigma}(z) \leq & 2 C_{\sigma} \sum_{j, m: \lambda_{j} \leq z} \sum_{m=1}^{\infty}\left(z-\lambda_{j}\right)_{+}^{\sigma-1} T_{\alpha j m} \\
& +2 R(\sigma, \alpha, z)
\end{aligned}
$$

where

$$
\begin{aligned}
& R(\sigma, \alpha, z) \\
& =\sum_{j, q: \lambda_{j} \leq z<\lambda_{q}} T_{\alpha j q}\left(z-\lambda_{j}\right)_{+}^{\sigma-1} \frac{\left(z-\lambda_{j}\right)-C_{\sigma}\left(\lambda_{q}-\lambda_{j}\right)}{\lambda_{q}-\lambda_{j}} .
\end{aligned}
$$

We average over $\alpha=1, \cdots, n$ in (3.5),

$$
\begin{gathered}
n R_{\sigma}(z) \leq 2 C_{\sigma} \sum_{j=1}^{\infty}\left(z-\lambda_{j}\right)_{+}^{\sigma-1} \lambda_{j}+2 \sum_{\alpha=1}^{n} R(\sigma, \alpha, z) \\
\text { Since } R_{\sigma}(z)=\sum_{k=1}^{\infty}\left(z-\lambda_{k}\right)_{+}^{\sigma} \text {, and } R_{\sigma}^{\prime}(z)=\sigma R_{\sigma-1}(z) \text {, } \\
\sum_{j=1}^{\infty}\left(z-\lambda_{j}\right)_{+}^{\sigma-1} \lambda_{j}=z R_{\sigma-1}(z)-R_{\sigma}(z)
\end{gathered}
$$

By (3.6) and (3.7),

$$
R_{\sigma}(z) \leq \frac{2 C_{\sigma}}{n}\left[z R_{\sigma-1}(z)-R_{\sigma}(z)\right]+\frac{2}{n} \sum_{\alpha=1}^{n} R(\sigma, \alpha, z)
$$

and then 
$\left[\left(1+\frac{2 C_{\sigma}}{n}\right) R_{\sigma}(z)-\frac{2 C_{\sigma} z}{n} R_{\sigma-1}(z)\right] \leq \frac{2}{n} \sum_{\alpha=1}^{n} R(\sigma, \alpha, z)($

Now we separate into three cases.

Case 1. $1 \leq \sigma \leq 2$. In this case $C_{\sigma}=1$ and

$$
\begin{aligned}
& R(\sigma, \alpha, z) \\
& =\sum_{j, q: \lambda_{j} \leq z<\lambda_{q}} T_{\alpha j q}\left(z-\lambda_{j}\right)_{+}^{\sigma-1}\left(\frac{\left(z-\lambda_{j}\right)-\left(\lambda_{q}-\lambda_{j}\right)}{\lambda_{q}-\lambda_{j}}\right) \leq 0
\end{aligned}
$$

then $\left(1+\frac{2}{n}\right) R_{\sigma}(z)-\frac{2 z}{n} R_{\sigma-1}(z) \leq 0$ which is (3.1).

Since $R_{\sigma}^{\prime}(z)=\sigma R_{\sigma-1}(z)$, substituting it to (3.1), we can get (3.2).

Case 2. $0<\sigma<1$. Since the sum defining $R(\sigma, \alpha, z)$ runs over $\lambda_{j} \leq z<\lambda_{q}$,

$$
\begin{aligned}
\frac{\left(z-\lambda_{j}\right)-C_{\sigma}\left(\lambda_{q}-\lambda_{j}\right)}{\lambda_{q}-\lambda_{j}} & \leq \frac{\left(\lambda_{q}-\lambda_{j}\right)-C_{\sigma}\left(\lambda_{q}-\lambda_{j}\right)}{\lambda_{q}-\lambda_{j}} \\
& =1-C_{\sigma}=1-\frac{\sigma}{2}>0
\end{aligned}
$$

Therefore

$$
\begin{aligned}
\sum_{\alpha=1}^{n} R(\sigma, \alpha, z) & \leq\left(1-C_{\sigma}\right) \sum_{j=1}^{\infty}\left(z-\lambda_{j}\right)_{+}^{\sigma-1} \lambda_{j} \\
& =\left(1-C_{\sigma}\right)\left(z R_{\sigma-1}(z)-R_{\sigma}(z)\right)
\end{aligned}
$$

Substituting this into (3.8), we have

$$
\frac{2 z}{n} R_{\sigma-1}(z) \geq\left(1+\frac{2}{n}\right) R_{\sigma}(z)
$$

which is equivalent to (3.1), also we can get (3.2).

On the other hand

$$
\left(\frac{R_{\sigma}(z)}{z^{\sigma\left(1+\frac{n}{2}\right)}}\right)^{\prime}=\frac{R_{\sigma}^{\prime}(z) z^{\sigma\left(1+\frac{n}{2}\right)}-R_{\sigma}(z) \sigma\left(1+\frac{n}{2}\right) z^{\sigma\left(1+\frac{n}{2}\right)-1}}{z^{2 \sigma\left(1+\frac{n}{2}\right)}}
$$

$\geq 0$

thus $\frac{R_{\sigma}(z)}{z^{\sigma\left(1+\frac{n}{2}\right)}}$ is a nondecreasing function with respect to $Z$.

Case 3. $\sigma \geq 2$. Similar to case 2 , we obtain

$$
R(\sigma, \alpha, z) \leq\left(1-C_{\sigma}\right) \sum_{\lambda_{j} \leq z<\lambda_{q}} T_{\alpha j q}\left(z-\lambda_{j}\right)_{+}^{\sigma-1}
$$

but now $1-C_{\sigma}=1-\frac{\sigma}{2}<0$, then $R(\sigma, \alpha, z) \leq 0$. Substituting it into (3.8),

$$
\left(1+\frac{2 C_{\sigma}}{n}\right) R_{\sigma}(z)-\frac{2 C_{\sigma} z}{n} R_{\sigma-1}(z) \leq 0
$$

(3.3)

Then we have $R_{\sigma-1}(z) \geq\left(1+\frac{n}{\sigma}\right) \frac{1}{z} R_{\sigma}(z)$, which is
(3.3).

Since $R_{\sigma}^{\prime}(z)=\sigma R_{\sigma-1}(z)$, we have

$R_{\sigma}^{\prime}(z) \geq(\sigma+n) \frac{1}{z} R_{\sigma}(z)$, which is (3.4).

Similarly

$$
\begin{aligned}
\left(\frac{R_{\sigma}(z)}{z^{\sigma+n}}\right)^{\prime} & =\frac{R_{\sigma}^{\prime}(z) z^{\sigma+n}-R_{\sigma}(z)(\sigma+n) z^{\sigma+n-1}}{z^{2(\sigma+n)}} \\
& \geq 0
\end{aligned}
$$

thus $\frac{R_{\sigma}(z)}{z^{\sigma+n}}$ is a nondecreasing function with respect to $z$. This completes the proof of the theorem.

\section{Acknowledgements}

This work was supported by Shanghai Leading Academic Discipline Project (S30501) and Innovation Programm of Shanghai Municipal Education Commission (10ZZ93).

\section{References}

[1] G. N. Hile and M. H. Protter, "Inequalities for Eigenvalues of the Laplacian," Indiana University Mathematics Journal, Vol. 29, No. 4, 1980, pp. 523-538. doi:10.1512/iumj.1980.29.29040

[2] E. M. Harrell II and J. Stubbe, “On Trace Identities and Universal Eigenvalue Estimates for Some Partial Differential Operators," Transactions of the American Mathematical Society, Vol. 349, No. 5, 1997, pp. 1797-1809. doi:10.1090/S0002-9947-97-01846-1

[3] E. M. Harrell and L. Hermi, "Differential Inequalities for Riesz Means and Weyl-Type Bounds for Eigenvalues," Journal of Functional Analysis, Vol. 254, No. 12, 2008, pp. 3173-3191. doi:10.1016/j.jfa.2008.02.016

[4] A. El Soufi, E. M. Harrell and S. Ilias, "Universal Inequalities for the Eigenvalues of Laplace and Schröinger Operators on Submanifolds,” American Mathematical Society, Providence, Vol. 361, 2009, pp. 2337-2350.

[5] M. S. Ashbaugh and L. Hermi, "Universal Inequalities for Higher-Order Elliptic Operators," Proceedings of the American Mathematical Society, Vol. 126, 1998, pp. 2623-2630. doi:10.1090/S0002-9939-98-04707-8

[6] P. Lévy-Bruhl, “Résolubilité Locale et Globale d’Opérateurs Invariants du Second Ordre sur des Groupes de Lie Nilpotents,” Bulletin des Sciences Mathematiques, Vol. 104, 1980, pp. 369-391.

[7] D. Müller and M. M. Peloso, "Non-Solvability for a Class of Left-Invariant Second-Order Differential Operators on the Heisenberg Group," Transactions of the American 
Mathematical Society, Vol. 355, No. 5, 2003, pp. 20472064. doi:10.1090/S0002-9947-02-03232-4

[8] P. C. Niu and H. Q. Zhang, "Payne-Polya-Weinberge Type Inequalities for Eigenvalues of Nonelliptic Operators," Pacific Journal of Mathematics, Vol. 208, No. 2, 2003, pp. 325-345. doi:10.2140/pjm.2003.208.325

[9] G. Jia, "On Some Function Spaces and Variational Problems Related to the Heisenberg Group,” Ph.D. Thesis, Nanjing University of Science and Technology, Nanjing, 2003.
[10] M. S. Ashbaugh and L. Hermi, "A Unified Approach to Universal Inequalities for Eigenvalues of Elliptic Operators,” Pacific Journal of Mathematics, Vol. 217, No. 2, 2004, pp. 201-220. doi:10.2140/pjm.2004.217.201

[11] E. M. Harrell and L. Hermi, "On Riesz Means of Eigenvalues,” Eprint arXiv: 0712. 4088.

[12] B. Helffer and D. Robert, "Riesz Means of Bounded States and Semi-Classical Limit Connected with a Lieb-Thirring Conjecture, II,” Annales de l'I.H.P., Physique Théorique, Vol. 53, 1990, pp. 139-147. 